\title{
Structure of the space of states in RSOS models
}

\author{
Michio Jimbo ${ }^{a}$, Tetsuji Miwa ${ }^{b}$ and Yasuhiro Ohta ${ }^{c}$ \\ ${ }^{a}$ Department of Mathematics,Faculty of Science, \\ Kyoto University, Kyoto 606, Japan \\ ${ }^{b}$ Research Institute for Mathematical Sciences, \\ Kyoto University, Kyoto 606, Japan \\ ${ }^{c}$ Faculty of Engineering, \\ Hiroshima University, Higashi Hiroshima, Hiroshima 724 , Japan
}

\begin{abstract}
The restricted solid-on-solid models in the anti-ferromagnetic regime is studied in the framework of quantum affine algebras. Following the line developed recently for vertex models, a representation theoretical picture is presented for the structure of the space of states. The local operators and the creation/annihilation operators of quasi-particles are defined using vertex operators, and their commutation relations are calculated.
\end{abstract}




\section{$\S 0$. Introduction}

Representation theory of quantized affine algebras has brought several effective constructions of solutions to the Yang-Baxter equation (see e.g. [1-3]). It is more than natural to expect that, in solving the lattice models associated with the latter, the quantized affine algebras would offer essential information. The first step in this direction is to understand the structure of the space of states, or the 'Hilbert space', of the model in terms of representation theory. By space of states we mean the one spanned by finite excitations over the ground states.

Such an approach was launched in [4] for the XXZ model

$$
H_{X X Z}=-\frac{1}{2} \sum_{k=-\infty}^{\infty}\left(\sigma_{k+1}^{x} \sigma_{k}^{x}+\sigma_{k+1}^{y} \sigma_{k}^{y}+\Delta \sigma_{k+1}^{z} \sigma_{k}^{z}\right)
$$

in the anti-ferroelectric regime $\Delta<-1$. The $\sigma_{k}^{x}, \sigma_{k}^{y}, \sigma_{k}^{z}$ are the Pauli matrices acting on the $k$-th component of the tensor space $\cdots V \otimes V \otimes V \otimes \cdots\left(V=\mathbf{C}^{2}\right)$. Suggested by the corner transfer matrix method [5-7] and the crystal base theory [8], it was proposed [4] that the space of states is the level 0 module over the quantized affine algebra $U_{q}\left(\widehat{\mathfrak{s l}}_{2}\right)$

$$
V\left(\Lambda_{i}\right) \otimes V\left(\Lambda_{j}\right)^{*} \simeq \operatorname{Hom}_{\mathbf{C}}\left(V\left(\Lambda_{j}\right), V\left(\Lambda_{i}\right)\right)
$$

Here $V\left(\Lambda_{i}\right)$ denotes the level one irreducible highest weight module and $V\left(\Lambda_{i}\right)^{*}$ is its dual module. The choice of highest weight $\Lambda_{i}$ (resp. $\Lambda_{j}$ ) accomodates the boundary condition to the left (resp. right) end of the lattice. (To be precise, in (0.2) an appropriate completion of the tensor product is necessary [4].) Besides the manifest symmetry under $U_{q}\left(\widehat{\mathfrak{s l}}_{2}\right)$, an important feature of $(0.2)$ is that the local structure of the lattice is automatically coded therein. This statement is based on the fact that after $q$-adic completion the vertex operators provide isomorphisms $V\left(\Lambda_{i}\right) \rightarrow V\left(\Lambda_{i+1}\right) \otimes V$ ( $\operatorname{suffix} i$ to be read modulo 2). Iterating these one may 'insert' the finite tensor product $V^{\otimes n}$ in $(0.2)$

$$
V\left(\Lambda_{i}\right) \otimes V\left(\Lambda_{j}\right)^{*} \stackrel{\sim}{\longrightarrow} V\left(\Lambda_{i+n}\right) \otimes V^{\otimes n} \otimes V\left(\Lambda_{j}\right)^{*}
$$

and vice versa. This makes it possible to describe the local spin operators and their correlation functions in terms of vertex operators [9]. Analogous construction is discussed in [10] for the case of higher level representations of $U_{q}\left(\widehat{\mathfrak{s l}}_{2}\right)$.

The aim of this note is to extend the above framework to incorporate the restricted solid-on-solid (RSOS) models. Suggested by the relevant results from the corner transfer matrix $[6,11]$ and the crystal base theory [12], we postulate that the 'Hilbert space' in this case is the space of $U^{\prime}$-linear maps

$$
\operatorname{Hom}_{U^{\prime}}(V(\tilde{\xi}) \otimes V(\tilde{\eta}), V(\xi) \otimes V(\eta))
$$

where $U^{\prime}$ is the subalgebra of $U_{q}\left(\widehat{\mathfrak{s l}}_{2}\right)$ 'with the scaling element $d$ being dropped' [4]. Like in $(0.2)$, the pair $(\xi, \eta)$ (resp. $(\tilde{\xi}, \tilde{\eta})$ ) corresponds to the choice of the boundary condition to the left (resp. right) end of the lattice. We shall show that the local structure of the lattice and the creation/annihilation of elementary excitations can be formulated using vertex operators. For definiteness' sake we shall concentrate on the case of $U_{q}\left(\widehat{\mathfrak{s l}}_{2}\right)$ in this paper. Evidently the same scheme applies to the general case.

It should be mentioned that closely related structures appear in the literature on massive deformations of conformal field theory $[13,14]$. Some of the formulas in this 
paper parallel those of the deformation of the coset theory described in [15]. However the precise connection is yet to be uncovered.

The text is organized as follows. To fix the notations we recall in Sect. 1 some relevant facts about the vertex operators. In Sect. 2, we define the space of states of the RSOS models. In Sect. 3 the transfer matrix, creation and annihilation operators are introduced and their commutation relations are calculated. In Appendix 1, restricting to the case of the Ising model we will calculate the eigenvectors of the transfer matrix by means of the vertex operators to a few order in the $q$-series expansion. Appendix 2 contains the explicit formulas for the connection coefficients.

\section{$\S 1$. Vertex operators}

1.1 Notations Unless stated otherwise, we shall retain the notations of [4], such as $\Lambda_{i}, \alpha_{i}, h_{i}(i=0,1), c, d, \delta=\alpha_{0}+\alpha_{1}, P=\mathbf{Z} \Lambda_{0} \oplus \mathbf{Z} \Lambda_{1} \oplus \mathbf{Z} \delta, P^{*}=\mathbf{Z} h_{0} \oplus \mathbf{Z} h_{1} \oplus \mathbf{Z} d$ and $\rho=\Lambda_{0}+\Lambda_{1}$. One change from [4] is that we normalize the invariant form $($,$) on P$ as $\left(\alpha_{i}, \alpha_{i}\right)=2$. We identify $P^{*}$ with a subset of $P$ via $($,$) . For \lambda=m_{0} \Lambda_{0}+m_{1} \Lambda_{1}+n \delta$ we put $\bar{\lambda}=m_{1} \bar{\Lambda}_{1}, \bar{\Lambda}_{1}=\Lambda_{1}-\Lambda_{0}$. The quantized affine algebra $U=U_{q}\left(\widehat{\mathfrak{s l}}_{2}\right)$ is defined by generators $e_{i}, f_{i}, t_{i}=q^{h_{i}}(i=0,1)$ and $q^{d}$ over $F=\mathbf{Q}(q)$, and $U^{\prime}=U_{q}^{\prime}\left(\widehat{\mathfrak{s l}}_{2}\right)$ signifies the subalgebra generated by $e_{i}, f_{i}$ and $t_{i}(i=0,1)$. The comultiplication $\Delta$ and the antipode $a$ are as in [4].

Set $P_{k}=\left\{\lambda \in P \mid\left\langle h_{i}, \lambda\right\rangle \in \mathbf{Z}_{>0},\langle c, \lambda\rangle=k\right\}, P_{k}^{0}=\left\{\lambda \in P_{k} \mid\langle d, \lambda\rangle=0\right\}$. We denote by $V(\lambda)$ the irreducible module with highest weight $\lambda \in P_{k}$ and a fixed highest weight vector $u_{\lambda} \in V(\lambda)_{\lambda}$. The weight space is denoted by $V(\lambda)_{\nu}(\nu \in P)$.

$\left.v_{N+1}^{(N)}=0\right)$

Let $V^{(N)}=\oplus_{j=0}^{N} F v_{j}^{(N)}$ denote the $(N+1)$-dimensional $U^{\prime}$-module given by $\left(v_{-1}^{(N)}=\right.$

$$
\begin{aligned}
& e_{1} v_{j}^{(N)}=[j] v_{j-1}^{(N)}, \quad f_{1} v_{j}^{(N)}=[N-j] v_{j+1}^{(N)}, \quad t_{1} v_{j}^{(N)}=q^{N-2 j} v_{j}^{(N)}, \\
& e_{0}=f_{1}, \quad f_{0}=e_{1}, \quad t_{0}=t_{1}^{-1} \quad \text { on } V^{(N)} .
\end{aligned}
$$

In the case $N=1$ we shall also write $v_{+}=v_{0}^{(1)}$ and $v_{-}=v_{1}^{(1)}$. Both $V^{(N)}$ and $V^{(1)}$ will play a role in the sequel. Let $V$ be a finite dimensional $U^{\prime}$-module. As in [8] its affinization means the $U$-module structure on $V_{z}=V \otimes F\left[z, z^{-1}\right]$ given as follows.

$$
\begin{aligned}
& e_{i}\left(v \otimes z^{n}\right)=\left(e_{i} v\right) \otimes z^{n+\delta_{i 0}}, \quad\left(f_{i} v \otimes z^{n}\right)=\left(f_{i} v\right) \otimes z^{n-\delta_{i 0}}, \\
& t_{i}\left(v \otimes z^{n}\right)=\left(t_{i} v\right) \otimes z^{n}, \quad q^{d}\left(v \otimes z^{n}\right)=v \otimes(q z)^{n} .
\end{aligned}
$$

For a $U$-(or $U^{\prime}$-)module $M$ the dual module $M^{* a^{ \pm 1}}$ is defined to be the restricted dual space $M^{*}=\oplus_{\nu} M_{\nu}^{*}$ endowed with the left module structure via $a^{ \pm 1}$ ( $a$ : antipode)

$$
\langle x u, v\rangle=\left\langle u, a^{ \pm 1}(x) v\right\rangle \quad \text { for } \quad x \in U, u \in M^{*}, v \in M
$$

The affinization $\left(V^{* a^{ \pm 1}}\right)_{z}$ can be canonically identified with the dual $\left(V_{z}\right)^{* a^{ \pm 1}}$ in the above sense via the pairing $\left\langle u \otimes z^{m}, v \otimes z^{n}\right\rangle=\langle u, v\rangle \delta_{m+n 0}\left(u \in V^{*}, v \in V\right)$. The modules $V_{z}^{(N)}$ are self-dual in the sense that there exist the following isomorphisms of $U$-modules

$$
C_{ \pm}^{(N)}: V_{z q^{\mp 2}}^{(N)} \stackrel{\sim}{\longrightarrow} V_{z}^{(N) * a^{ \pm 1}}, \quad C_{ \pm}^{(N)} v_{j}^{(N)}=(-1)^{j} q^{-j(N-j \mp 1)}\left[\begin{array}{c}
N \\
j
\end{array}\right]^{-1} v_{N-j}^{(N) *}
$$


Here $\left\{v_{j}^{(N) *}\right\}$ signifies the dual base of $\left\{v_{j}^{(N)}\right\}$ and (1.2) is to be extended $F\left[z, z^{-1}\right]$ linearly. For $N=1(1.2)$ simplifies to $C_{ \pm}^{(1)} v_{+}=v_{-}^{*}, C_{ \pm}^{(1)} v_{-}=-q^{ \pm 1} v_{+}^{*}$.

1.2 Vertex operators We recall below the properties of the vertex operators (VOs) relevant to the subsequent discussions. For more details see [4,12].

Fix positive integers $k, N$ such that $k \geq N$, and let $\lambda, \mu \in P_{k}^{0}$. We set $\Delta_{\lambda}=$ $(\lambda, \lambda+2 \rho) / 2(k+2)$. We shall use the following types of VOs:

Type $I$

$$
\begin{aligned}
& \Phi_{\lambda}^{\mu V^{(N)}}(z)=z^{\Delta_{\mu}-\Delta_{\lambda}} \widetilde{\Phi}_{\lambda}^{\mu V^{(N)}}(z), \quad \widetilde{\Phi}_{\lambda}^{\mu V^{(N)}}(z): V(\lambda) \longrightarrow V(\mu) \widehat{\otimes} V_{z}^{(N)}, \\
& \Phi_{\mu V^{(N)}}^{\lambda}(z)=z^{\Delta_{\lambda}-\Delta_{\mu}} \widetilde{\Phi}_{\mu V^{(N)}}^{\lambda}(z), \quad \widetilde{\Phi}_{\mu V^{(N)}}^{\lambda}(z): V(\mu) \otimes V_{z}^{(N)} \longrightarrow V(\lambda) \widehat{\otimes} F\left[z, z^{-1}\right],
\end{aligned}
$$

Type II

$$
\begin{aligned}
& \Phi_{\lambda}^{V^{(N)} \mu}(z)=z^{\Delta_{\mu}-\Delta_{\lambda}} \widetilde{\Phi}_{\lambda}^{V^{(N)} \mu}(z), \quad \widetilde{\Phi}_{\lambda}^{V^{(N)} \mu}(z): V(\lambda) \longrightarrow V_{z}^{(N)} \widehat{\otimes} V(\mu), \\
& \Phi_{V^{(N)} \mu}^{\lambda}(z)=z^{\Delta_{\lambda}-\Delta_{\mu}} \widetilde{\Phi}_{V^{(N)} \mu}^{\lambda}(z), \quad \widetilde{\Phi}_{V^{(N)} \mu}^{\lambda}(z): V_{z}^{(N)} \otimes V(\mu) \longrightarrow V(\lambda) \widehat{\otimes} F\left[z, z^{-1}\right] .
\end{aligned}
$$

For example (1.3a) means a formal series of the form

$$
\widetilde{\Phi}_{\lambda}^{\mu V^{(N)}}(z)=\sum \Phi_{j n} \otimes v_{j}^{(N)} z^{-n}, \quad \Phi_{j n}: V(\lambda)_{\nu} \longrightarrow V(\mu)_{\nu-\mathrm{wt} v_{j}+n \delta} \quad \forall \nu
$$

such that it satisfies the intertwining relations

$$
\widetilde{\Phi}_{\lambda}^{\mu V^{(N)}}(z) \circ x=\Delta(x) \circ \widetilde{\Phi}_{\lambda}^{\mu V^{(N)}}(z) \quad \forall x \in U .
$$

Here in the right hand side the action of $U$ on $V$ is in the sense of (1.1). Likewise (1.3b) means a formal series of $F\left[z, z^{-1}\right]$-linear maps

$$
\widetilde{\Phi}_{\mu V^{(N)}}^{\lambda}(z)\left(\cdot \otimes v_{j}\right)=\sum \Phi_{j n}^{*} \otimes z^{-n}, \quad \Phi_{j n}^{*}: V(\mu)_{\nu} \longrightarrow V(\lambda)_{\nu+\mathrm{wt} v_{j}+n \delta} \quad \forall \nu
$$

that enjoys similar intertwining properties. We write $\widetilde{\Phi}_{\lambda}^{\mu V^{(N)}}=\widetilde{\Phi}_{\lambda}^{\mu V^{(N)}}(1)=\sum \Phi_{j n} \otimes$ $v_{j}^{(N)}$ to mean the corresponding intertwiner of $U^{\prime}$-modules $V(\lambda) \rightarrow \widehat{V}(\mu) \otimes V^{(N)}$ where $\widehat{V}(\mu)=\prod_{\nu} V(\mu)_{\nu}$. Similar convention is used for the other types of VOs.

1.3 Admissibility As in Sect. 1.2 we let $k \geq N$. The VOs $(1.3 a)$, (1.4a) are in one-toone correspondence with the vectors $v \in V^{(N)}$ such that [12]

$$
e_{i}^{\left\langle h_{i}, \mu\right\rangle+1} v=0 \quad(i=0,1), \quad \text { wt } v \equiv \lambda-\mu \bmod \delta .
$$

We say that a pair of weights $(\mu, \lambda) \in\left(P_{k}^{0}\right)^{2}$ is $V^{(N)}$-admissible if (1.7) admits a nontrivial solution $v$ [12]. Since $V^{(N)}$ is multiplicity free, there exists at most one such $v$ up to proportionality. If $\lambda=(k-a) \Lambda_{0}+a \Lambda_{1}$ and $\mu=(k-b) \Lambda_{0}+b \Lambda_{1}$, then a nontrivial solution of (1.7) exists if and only if

$$
a-b \in\{N, N-2, \cdots,-N\}, \quad N \leq a+b \leq 2 k-N .
$$


In the special case $N=1$ this means that either $b=a+1$ with $a<k$, or $b=a-1$ with $a>0$. VOs as

Choosing the scalar multiple of $v$ (1.7) appropriately we normalize the corresponding

$$
\begin{aligned}
& \widetilde{\Phi}_{\lambda}^{\mu V^{(N)}}(z) u_{\lambda}=u_{\mu} \otimes v_{j}^{(N)}+\cdots, \quad \widetilde{\Phi}_{\lambda}^{V^{(N)} \mu}(z) u_{\lambda}=v_{j}^{(N)} \otimes u_{\mu}+\cdots, \\
& \widetilde{\Phi}_{\mu V^{(N)}}^{\lambda}(z)\left(u_{\mu} \otimes v_{j}^{(N)}\right)=u_{\lambda}+\cdots, \quad \widetilde{\Phi}_{V^{(N)} \mu}^{\lambda}(z)\left(v_{j}^{(N)} \otimes u_{\mu}\right)=u_{\lambda}+\cdots,
\end{aligned}
$$

where $j$ is such that $\lambda \equiv \mu+(N-2 j) \bar{\Lambda}_{1} \bmod \delta$. In the first equation of $(1.9 a)$ the symbol $\cdots$ indicates terms in $V(\mu)_{\nu} \otimes V^{(N)}$ with $\nu \neq \mu$. Similarly for the other cases.

Remark 1. For any $U^{\prime}$-modules $M, N, \cdots$ we have canonical isomorphisms

$$
\begin{aligned}
& \operatorname{Hom}_{U^{\prime}}\left(M_{1} \otimes M_{2}, N\right) \stackrel{\sim}{\longrightarrow} \operatorname{Hom}_{U^{\prime}}\left(M_{2}, M_{1}^{* a^{-1}} \otimes N\right), \\
& \operatorname{Hom}_{U^{\prime}}\left(M_{1} \otimes M_{2}, N\right) \stackrel{\sim}{\longrightarrow} \operatorname{Hom}_{U^{\prime}}\left(M_{1}, N \otimes M_{2}^{* a}\right) .
\end{aligned}
$$

Accordingly $\widetilde{\Phi}_{\mu V^{(N)}}^{\lambda}(z)$ gives rise to the VO

$$
\begin{aligned}
& \widetilde{\Phi}_{\mu}^{\lambda V^{(N) * a}}(z): V(\mu) \longrightarrow V(\lambda) \otimes V^{(N) * a}, \\
& \widetilde{\Phi}_{\mu}^{\lambda V^{(N) * a}}(z)=\sum_{j, n} \Phi_{j n}^{*} \otimes v_{j}^{*(N)}
\end{aligned}
$$

where $\Phi_{j n}^{*}$ is given in (1.6). The normalization (1.9b) implies $\widetilde{\Phi}_{\mu}^{\lambda V^{(N) * a}}(z) u_{\mu}=u_{\lambda} \otimes$ $v_{j}^{(N) *}+\cdots$. Likewise $\widetilde{\Phi}_{V^{(N)} \mu}^{\lambda}(z)$ gives rise to $\widetilde{\Phi}_{\mu}^{V^{(N) * a^{-1}} \lambda}(z)$.

Remark 2. Let us call a (possibly infinite) sequence $\left(\mu_{j}\right)_{j}$ of weights admissible if each $\left(\mu_{j}, \mu_{j+1}\right)$ is. Then, for each $n \geq 1$ and $\lambda, \lambda^{\prime}$, the space of vertex operators $V(\lambda) \rightarrow$ $\widehat{V}\left(\lambda^{\prime}\right) \otimes\left(V^{(N)}\right)^{\otimes n}$ has the basis

$$
\widetilde{\Phi}_{\mu_{1}}^{\lambda^{\prime} V^{(N)}} \circ \cdots \circ \widetilde{\Phi}_{\lambda}^{\mu_{n-1} V^{(N)}}, \quad\left(\lambda^{\prime}, \mu_{1}, \cdots, \mu_{n-1}, \lambda\right): \text { admissible. }
$$

1.4 Crystals The representation $V^{(N)}$ has an upper crystal base $\left(L^{(N)}, B^{(N)}\right)$ given by $L^{(N)}=\oplus_{j=0}^{N} A v_{j}^{(N)}, B^{(N)}=\left\{v_{j} \bmod q L^{(N)}\right\}_{0 \leq j \leq N}$ where $A=\{f(q) \in F \mid f(q)$ has no pole at $q=0\}$. Let $L(\lambda)$ denote the (upper) crystal lattice of $V(\lambda)$. The type I VOs preserve the crystal lattice [12] in the sense $\widetilde{\Phi}_{\lambda}^{\mu V^{(N)}}(L(\lambda)) \subset \widehat{L}(\mu) \otimes L^{(N)}$, where $\widehat{L}(\mu)=\prod_{\nu} L(\mu)_{\nu}$. It is known that

$$
\Phi_{\mu V^{(N)}}^{\lambda}(z) \circ \Phi_{\lambda}^{\mu V^{(N)}}(z)=g_{\lambda}^{\mu} \times \operatorname{id}_{V(\lambda)}
$$

with a scalar $g_{\lambda}^{\mu}=g_{\lambda}^{\mu}(k, N)[12]$. The formula for $g_{\lambda}^{\mu}(k, 1)$ is given in Appendix 2, $(A 2.3)$.

The crystal $B^{(N)}$ is perfect of level $N$ in the sense of [8]. This implies that for each $\eta \in P_{N}^{0}$ of level $N$ there are unique weights $\hat{\eta}, \hat{\eta}^{\prime}$ such that the pairs $(\eta, \hat{\eta}),\left(\hat{\eta}^{\prime}, \eta\right)$ are admissible. In our case we have $\hat{\eta}=\hat{\eta}^{\prime}=\sigma(\eta)$ where $\sigma$ denotes the involution

$$
\sigma\left((N-a) \Lambda_{0}+a \Lambda_{1}\right)=a \Lambda_{0}+(N-a) \Lambda_{1} .
$$


If $\lambda=\eta$ and $\mu=\sigma(\eta)$ have level $N$, then we have in addition to (1.11)

$$
\Phi_{\eta}^{\sigma(\eta) V^{(N)}}(z) \circ \Phi_{\sigma(\eta) V^{(N)}}^{\eta}(z)=g_{\eta}^{\sigma(\eta)} \times \operatorname{id}_{V(\hat{\eta}) \otimes V^{(N)}}
$$

This shows that (after completion) the VO provides with an isomorphism $\widetilde{\Phi}_{\eta}^{\sigma(\eta) V^{(N)}}$ : $V(\eta) \longrightarrow V(\sigma(\eta)) \otimes V^{(N)}$.

1.5 Connection coefficients Let $\bar{R}_{N N}\left(z_{1} / z_{2}\right) \in \operatorname{End}\left(V_{z_{1}}^{(N)} \otimes V_{z_{2}}^{(N)}\right)$ be the $R$ matrix

$$
P \bar{R}_{N N}\left(z_{1} / z_{2}\right) \Delta(x)=\Delta(x) P \bar{R}_{N N}\left(z_{1} / z_{2}\right) \quad \forall x \in U, \quad P u \otimes v=v \otimes u
$$

normalized by $\bar{R}_{N N}\left(z_{1} / z_{2}\right) v_{0}^{(N)} \otimes v_{0}^{(N)}=v_{0}^{(N)} \otimes v_{0}^{(N)}$. Let further $\lambda, \mu, \nu \in P_{k}^{0}$. Then the following commutation relation takes place among the VOs [3]:

$$
\begin{aligned}
& \bar{R}_{N N}\left(z_{1} / z_{2}\right) \Phi_{\mu}^{\nu V_{1}^{(N)}}\left(z_{1}\right) \Phi_{\lambda}^{\mu V_{2}^{(N)}}\left(z_{2}\right) \\
& \quad=\sum_{\mu^{\prime}} \Phi_{\mu^{\prime}}^{\nu V^{(N)}}\left(z_{2}\right) \Phi_{\lambda}^{\mu^{\prime} V_{1}^{(N)}}\left(z_{1}\right) \times W_{k}^{N}\left(\begin{array}{cc}
\lambda & \mu \\
\mu^{\prime} & \nu
\end{array} \mid z_{1} / z_{2}\right) .
\end{aligned}
$$

Here if $\Phi_{\mu}^{\nu V^{(N)}}(z)=\sum_{j} \Phi_{\mu j}^{\nu V^{(N)}}(z) \otimes v_{j}^{(N)}$ then we write

$$
\begin{aligned}
& \Phi_{\mu}^{\nu V_{1}^{(N)}}\left(z_{1}\right) \Phi_{\lambda}^{\mu V_{2}^{(N)}}\left(z_{2}\right)=\sum_{j j^{\prime}} \Phi_{\mu j}^{\nu V^{(N)}}\left(z_{1}\right) \Phi_{\lambda j^{\prime}}^{\mu V^{(N)}}\left(z_{2}\right) \otimes v_{j}^{(N)} \otimes v_{j^{\prime}}^{(N)} \\
& \Phi_{\mu^{\prime}}^{\nu V^{(N)}}\left(z_{2}\right) \Phi_{\lambda}^{\mu^{\prime} V_{1}^{(N)}}\left(z_{1}\right)=\sum_{j j^{\prime}} \Phi_{\mu^{\prime} j^{\prime}}^{\nu V^{(N)}}\left(z_{2}\right) \Phi_{\lambda j}^{\mu^{\prime} V^{(N)}}\left(z_{1}\right) \otimes v_{j}^{(N)} \otimes v_{j^{\prime}}^{(N)}
\end{aligned}
$$

The connection coefficients

$$
W_{k}^{N}\left(\begin{array}{cc|c}
\lambda & \mu \\
\mu^{\prime} & \nu & z_{1} / z_{2}
\end{array}\right)
$$

satisfy the Yang-Baxter equation in the face formulation. Their explicit form for $N=1$ is included in the Appendix 2, $(A 2.1, A 2.2)$. 


\section{$\S 2$. Space of states in the RSOS models}

2.1 RSOS models Let us recall the basic features of the solvable RSOS models associated with $U_{q}\left(\widehat{\mathfrak{s l}}_{2}\right)[6,11]$. The model is labeled by a pair of positive integers $(l, N)$, $l=k-N$. At each lattice site $(i, j) \in \mathbf{Z}^{2}$ one places a random variable $\sigma_{i j}$ taking values in the set of local states $S=\{0,1, \cdots, k\}$. One imposes further a restriction that for all $i, j$ the configurations on the adjacent lattice sites $\left(\sigma_{i j}, \sigma_{i+1}\right),\left(\sigma_{i j+1}, \sigma_{i j}\right)$ be admissible in the sense of (1.8). We identify an element $a \in S$ with the level $k$ dominant integral weight $\lambda_{a}=(k-a) \Lambda_{0}+a \Lambda_{1} \in P_{k}^{0}$. The Boltzmann weight of the model is attached to each configuration $\left(\lambda, \mu, \nu, \mu^{\prime}\right)$ on the NW, NE, SE, SW corners of an elementary face, and is given by the connection coefficients (1.14).

We shall consider exclusively the anti-ferromagnetic regime (regime III of [11]). In the present parametrization this reads

$$
0<q^{2}<1, \quad 1<z<q^{-2} \text {. }
$$

Consider the limit of extreme order $q=0$. In this limit only those configurations that are invariant under the shift in the NE-SW direction survive. Hence they can be labeled by configurations in the horizontal direction. The ground states are given by the sequences of states of the form $\cdots b c b c b c \cdots$ with admissible $(b, c) \in S \times S$. They correspond bijectively to a pair of dominant integral weights $(\xi, \eta) \in P_{l}^{0} \times P_{N}^{0}$ of levels $l$ and $N$ respectively, via $\lambda_{b}=\xi+\eta, \lambda_{c}=\xi+\sigma(\eta)$. The excited states consist of admissible sequences ('restricted paths') $\left(a^{(j)}\right)_{j \in \mathbf{Z}}$ satisfying the boundary condition at the right (resp. left) end of the lattice specified by a ground state $(\tilde{\xi}, \tilde{\eta})(\operatorname{resp} .(\xi, \eta))$. This means that $a^{(j)}=\xi+\sigma^{j}(\eta)$ for $j \ll 0, a^{(j)}=\tilde{\xi}+\sigma^{j}(\tilde{\eta})$ for $j \gg 0$. (If we consider the periodic boundary condition then only those states with $\tilde{\xi}=\xi$ and $\tilde{\eta}=\eta$ appear.)

We wish to find the eigenstates of the row transfer matrix $T(z)$ at nonzero $q$. We expect that for $0<q^{2}<1$ these eigenstates can be expanded in powers of $q$, as an infinite sum of the restricted paths obeying the same boundary condition as the initial term $q=0$. In the sequel we shall present a mathematical definition of the space of eigenstates consistent with the naïve picture described above.

2.2 Space of states For $\xi \in P_{l}^{0}$ and $\eta \in P_{N}^{0}$, consider the tensor product

$$
V(\xi) \otimes V(\eta)=\oplus_{\lambda \in P_{k}^{0}} W(\xi \eta ; \lambda), \quad W(\xi \eta ; \lambda) \simeq \Omega_{\xi \eta \lambda} \otimes V(\lambda)
$$

where $\Omega_{\xi \eta \lambda}$ stands for the space of highest weight vectors

$$
\Omega_{\xi \eta \lambda}=\left\{v \in V(\xi) \otimes V(\eta) \mid e_{i} v=0, t_{i} v=q^{\left\langle h_{i}, \lambda\right\rangle} v \quad \forall i\right\}
$$

We regard $\Omega_{\xi \eta \lambda}$ as a $U$-module on which $U^{\prime}$ acts trivially and $q^{d}$ acts as $q^{d} \otimes q^{d}$ on $V(\xi) \otimes V(\eta)$. It has been known by the corner transfer matrix method [6], and later systematized by the crystal base theory [12], that one can label the base vectors of $\Omega_{\xi \eta \lambda}$ by restricted paths, by assigning suitable weights to the latter.

Now set

$$
\begin{aligned}
\mathcal{F}_{\xi \eta ; \tilde{\xi} \tilde{\eta}} & =\operatorname{Hom}_{U^{\prime}}(V(\tilde{\xi}) \otimes V(\tilde{\eta}), V(\xi) \otimes V(\eta)) \\
& =\oplus_{\lambda} \operatorname{Hom}_{U^{\prime}}(V(\lambda), V(\xi) \otimes V(\eta)) \otimes \operatorname{Hom}_{U^{\prime}}(V(\tilde{\xi}) \otimes V(\tilde{\eta}), V(\lambda))
\end{aligned}
$$

The second equality follows from the decomposition (2.1). To be precise, we need a completion of this space in the $q$-adic sense to accomodate the vertex operators which 
produce infinite sums. To simplify the presentation, we will not discuss this point. The symbols $\otimes$, etc. should be understood appropriately.

For each $n \geq 1$, let $\eta^{(n)}=\sigma^{n}(\eta)$. The iteration of (1.3) (with $z=1$ ) gives rise to

$$
\Phi^{(n)}: V(\eta) \stackrel{\sim}{\longrightarrow} V\left(\eta^{(n)}\right) \otimes\left(V^{(N)}\right)^{\otimes n} .
$$

This enables us to make an identification

$$
\begin{aligned}
& \operatorname{Hom}_{U^{\prime}}(V(\lambda), V(\xi) \otimes V(\eta)) \\
& \quad \stackrel{\sim}{\longrightarrow} \operatorname{Hom}_{U^{\prime}}\left(V(\lambda), V(\xi) \otimes V\left(\eta^{(n)}\right) \otimes\left(V^{(N)}\right)^{\otimes n}\right) \\
& \stackrel{\sim}{\longrightarrow} \oplus_{\lambda^{\prime}} \operatorname{Hom}_{U^{\prime}}\left(V\left(\lambda^{\prime}\right), V(\xi) \otimes V\left(\eta^{(n)}\right)\right) \otimes \operatorname{Hom}_{U^{\prime}}\left(V(\lambda), V\left(\lambda^{\prime}\right) \otimes\left(V^{(N)}\right)^{\otimes n}\right)
\end{aligned}
$$

The first arrow is given by id $\otimes \Phi^{(n)}$, the second is given by the decomposition $(2.1)$.

We take (2.2) to be the mathematical definition of the space of states corresponding to the fixed boundary condition $(\xi, \eta)(\operatorname{resp} .(\tilde{\xi}, \tilde{\eta}))$ to the left (resp. right) half of the horizontal line. Because of the restriction condition in the model, horizontal configurations between sites separated by $n$ steps are admissible sequences $\left(\lambda^{\prime}, \mu_{1}, \cdots, \mu_{n-1}, \lambda\right)$. In view of (1.10) we can identify them with the base vectors of $\operatorname{Hom}_{U^{\prime}}\left(V(\lambda), V\left(\lambda^{\prime}\right) \otimes\left(V^{(N)}\right)^{\otimes n}\right)$. Therefore (2.4) means that the naïve local structure of the lattice can be embedded in the space $(2.2)$.

2.3 Vacuum and inner product In the space $\mathcal{F}_{\xi \eta ; \xi \eta}$ there is the unique canonical element, i.e. the identity. We call it the vacuum vector $|\operatorname{vac}\rangle_{\xi \eta}=$ id.

For $f \in \mathcal{F}_{\xi \eta ; \xi^{\prime} \eta^{\prime}}$ and $g \in \mathcal{F}_{\xi^{\prime} \eta^{\prime} ; \xi \eta}$ we define a bilinear pairing

$$
\langle f \mid g\rangle_{\xi \eta}=\frac{\operatorname{tr}_{V(\xi) \otimes V(\eta)}\left(q^{-2 \rho} f g\right)}{\operatorname{tr}_{V(\xi) \otimes V(\eta)}\left(q^{-2 \rho}\right)}=\sum_{\lambda} \frac{\chi_{\lambda}}{\chi_{\xi} \chi_{\eta}} \operatorname{tr}_{\Omega_{\xi \eta \lambda}}\left(q^{-2 \rho} f g\right)
$$

where

$$
\chi_{\lambda}=\operatorname{tr}_{V(\lambda)}\left(q^{-2 \rho}\right)=q^{-(2 \rho, \lambda)}(1+\cdots)
$$

signifies the prinicipally specialized character.

Remark. The scaling element $d$ has a well-defined action on $V(\xi) \otimes V(\eta)$ and normalizes the action of $U^{\prime}:\left[d, U^{\prime}\right] \subset U^{\prime}$. It follows that if $f \in \mathcal{F}_{\xi \eta ; \tilde{\xi} \tilde{\eta}}$ then $[d, f] \in \mathcal{F}_{\xi \eta ; \tilde{\xi} \tilde{\eta}}$. Hence $\mathcal{F}_{\xi \eta ; \tilde{\xi} \tilde{\eta}}$ admits a $\mathbf{Z}$-grading by $d$. 


\section{$\S 3$. Particles}

3.1 Translation In the same spirit as in [4] one may introduce the left translation operator by a lattice unit

$$
T=T_{\eta \tilde{\eta}}^{\sigma(\eta) \sigma(\tilde{\eta})}: \mathcal{F}_{\xi \eta ; \tilde{\xi} \tilde{\eta}} \longrightarrow \mathcal{F}_{\xi \sigma(\eta) ; \tilde{\xi} \sigma(\tilde{\eta})} .
$$

More generally we shall define $T(z)=T_{\eta \tilde{\eta}}^{\sigma(\eta) \sigma(\tilde{\eta})}(z)$ which is related to $T$ by

$$
T(z)=z^{-d} T(1) z^{d} .
$$

(Namely if $T=\sum T_{n}$ is the decomposition into the homogeneous components with respect to the grading via $d$, then $T(z)=\sum T_{n} z^{-n}$.) The image of a vector $f \in \mathcal{F}_{\xi \eta ; \tilde{\xi} \tilde{\eta}}$ by $T(z), T(z)(f) \in \mathcal{F}_{\xi \sigma(\eta) ; \tilde{\xi} \sigma(\tilde{\eta})}$, is defined to be the composition

$$
\begin{gathered}
V(\tilde{\xi}) \otimes V(\sigma(\tilde{\eta})) \stackrel{i d \otimes \Phi_{1}(z)}{\longrightarrow} V(\tilde{\xi}) \otimes V(\tilde{\eta}) \otimes V_{z}^{(N) * a^{-1}} \stackrel{f \otimes i d}{\longrightarrow} V(\xi) \otimes V(\eta) \otimes V_{z}^{(N) * a^{-1}} \\
\stackrel{i d \otimes \Phi_{2}(z)}{\longrightarrow} V(\xi) \otimes V(\sigma(\eta)) .
\end{gathered}
$$

We have set $\Phi_{1}(z)=\widetilde{\Phi}_{\sigma(\tilde{\eta})}^{\tilde{\eta} V^{(N) * a^{-1}}}(z)$ and $\Phi_{2}(z)=\Phi_{\eta V^{(N) * a-1}}^{\sigma(\eta)}(z)$. As in the vertex models it is natural to identify $z^{-d}$ with the corner transfer matrix. In view of (3.1) we identify $T(z)$ with the row transfer matrix of the RSOS model.

Remark. In $[4,10]$ the translation operator for the vertex models is formulated as the composition

$$
\begin{aligned}
& V(\lambda) \otimes V\left(\lambda^{\prime}\right)^{* a} \stackrel{\widetilde{\Phi}_{\lambda}^{\sigma(\lambda) V} \otimes i d}{\longrightarrow} V(\sigma(\lambda)) \otimes V \otimes V\left(\lambda^{\prime}\right)^{* a} \\
& \stackrel{i d \otimes \widetilde{\Phi}_{V \aleph^{\prime}}^{* \sigma\left(\lambda^{\prime}\right)}}{\longrightarrow} V(\sigma(\lambda)) \otimes V\left(\sigma\left(\lambda^{\prime}\right)\right)^{* a}
\end{aligned}
$$

where $V$ is perfect of level $k, \lambda, \lambda^{\prime}, \cdots \in P_{k}^{0}$, and $\widetilde{\Phi}_{V \lambda^{\prime}}^{* \sigma\left(\lambda^{\prime}\right)}: V \otimes V\left(\lambda^{\prime}\right)^{* a} \rightarrow V\left(\sigma\left(\lambda^{\prime}\right)\right)^{* a}$ is the transpose of $\widetilde{\Phi}_{\sigma\left(\lambda^{\prime}\right)}^{\lambda^{\prime} V^{* a}}: V\left(\sigma\left(\lambda^{\prime}\right)\right) \rightarrow V\left(\lambda^{\prime}\right) \otimes V^{* a^{-1}}$. Under the identification $V(\lambda) \otimes V\left(\lambda^{\prime}\right)^{* a} \simeq \operatorname{Hom}\left(V\left(\lambda^{\prime}\right), V(\lambda)\right)$, this means that $f \in \operatorname{Hom}\left(V\left(\lambda^{\prime}\right), V(\lambda)\right)$ is sent to the composition

$$
V\left(\sigma\left(\lambda^{\prime}\right)\right) \stackrel{\widetilde{\Phi}_{\sigma\left(\lambda^{\prime}\right)}^{\lambda a^{-1}}}{\longrightarrow} V\left(\lambda^{\prime}\right) \otimes V^{* a^{-1}} \stackrel{f \otimes i d}{\longrightarrow} V(\lambda) \otimes V^{* a^{-1}} \stackrel{\widetilde{\Phi}_{V(\lambda) V * a-1}^{\sigma(\lambda)}}{\longrightarrow} V(\sigma(\lambda)) .
$$

This can be compared with (3.2).

3.2 Creation and annihilation Let $\xi \in P_{l}^{0}, \eta \in P_{N}^{0}$, and let $\xi^{\prime} \in P_{l}^{0}, \eta^{\prime} \in P_{N}^{0}$ be such that $\left(\xi^{\prime}, \xi\right),\left(\eta^{\prime}, \eta\right)$ are $V^{(1)}$-admissible. We shall introduce $U$-linear operators

$$
\hat{\varphi}_{\xi \eta}^{* \xi^{\prime} \eta^{\prime}}(z), \hat{\varphi}_{\xi \eta}^{\xi^{\prime} \eta^{\prime}}(z): V(\xi) \otimes V(\eta) \longrightarrow V\left(\xi^{\prime}\right) \otimes V\left(\eta^{\prime}\right) .
$$

By definition $\hat{\varphi}_{\xi \eta}^{* \xi^{\prime} \eta^{\prime}}(z)$ is the composition

$$
\begin{aligned}
& V(\xi) \otimes V(\eta) \stackrel{\Phi_{\xi}^{\xi^{\prime} V^{(1)}}(z) \otimes \Phi_{\eta}^{V^{(1) * a-1}}}{\longrightarrow} \eta^{\prime}(z) \quad V\left(\xi^{\prime}\right) \otimes V_{z}^{(1)} \otimes V_{z}^{(1) * a^{-1}} \otimes V\left(\eta^{\prime}\right) \\
& i d \otimes\left\langle, \zeta \otimes i d \quad V\left(\xi^{\prime}\right) \otimes V\left(\eta^{\prime}\right) .\right.
\end{aligned}
$$


Similarly $\hat{\varphi}_{\xi \eta}^{\xi^{\prime} \eta^{\prime}}(z)$ is the composition

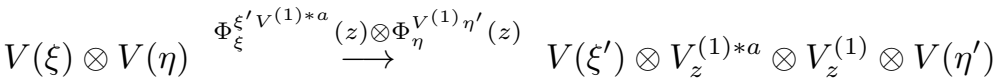

$$
\begin{aligned}
& i d \otimes\left\langle\underline{\langle}, \otimes i d \quad V\left(\xi^{\prime}\right) \otimes V\left(\eta^{\prime}\right) .\right.
\end{aligned}
$$

(Note that the canonical pairing $\langle\rangle:, V_{z}^{(1)} \otimes V_{z}^{(1) * a^{-1}} \rightarrow F$ or $V_{z}^{(1) * a} \otimes V_{z}^{(1)} \rightarrow F$ is $U$-linear.) In other words, we set

$$
\begin{aligned}
& \hat{\varphi}_{\xi \eta}^{* \xi^{\prime} \eta^{\prime}}(z)=\sum_{\varepsilon= \pm} \Phi_{\xi}^{\xi^{\prime} V^{(1)}}(z)_{\varepsilon} \otimes \Phi_{\eta}^{V^{(1) * a}} \eta^{-1}(z)_{\varepsilon} \\
& \hat{\varphi}_{\xi \eta}^{\xi^{\prime} \eta^{\prime}}(z)=\sum_{\varepsilon= \pm} \Phi_{\xi}^{\xi^{\prime} V^{(1) * a}}(z)_{\varepsilon} \otimes \Phi_{\eta}^{V^{(1)} \eta^{\prime}}(z)_{\varepsilon}
\end{aligned}
$$

where the components of the VOs are defined by

$$
\begin{aligned}
\Phi_{\xi}^{\xi^{\prime} V^{(1)}}(z) & =\sum_{\varepsilon} \Phi_{\xi}^{\xi^{\prime} V^{(1)}}(z)_{\varepsilon} \otimes v_{\varepsilon}, \quad \Phi_{\eta}^{V^{(1) * a-1} \eta^{\prime}}(z)=\sum_{\varepsilon} v_{\varepsilon}^{*} \otimes \Phi_{\eta}^{V^{(1) * a}{ }^{-1}} \eta^{\prime}(z)_{\varepsilon}, \\
\Phi_{\xi}^{\xi^{\prime} V^{(1) * a}}(z) & =\sum_{\varepsilon} \Phi_{\xi}^{\xi^{\prime} V^{(1) * a}}(z)_{\varepsilon} \otimes v_{\varepsilon}^{*}, \quad \Phi_{\eta}^{V^{(1)} \eta^{\prime}}(z)=\sum_{\varepsilon} v_{\varepsilon} \otimes \Phi_{\eta}^{V^{(1)} \eta^{\prime}}(z)_{\varepsilon},
\end{aligned}
$$

with $v_{\varepsilon}^{*}$ being the dual base of $v_{\varepsilon}$. In view of the isomorphism (1.2) we have the relation

$$
\hat{\varphi}_{\xi \eta}^{\xi^{\prime} \eta^{\prime}}(z)=\hat{\varphi}_{\xi \eta}^{* \xi^{\prime} \eta^{\prime}}\left(q^{-2} z\right) \times q^{2\left(\Delta_{\xi^{\prime}}+\Delta_{\eta^{\prime}}-\Delta_{\xi}-\Delta_{\eta}\right)}(-q) /\left(K_{\xi}^{\xi^{\prime}} K_{\eta}^{\eta^{\prime}}\right) .
$$

Here we have set $K_{\lambda}^{\mu}=-q$ if $\mu=\lambda+\bar{\Lambda}_{1},=1$ if $\mu=\lambda-\bar{\Lambda}_{1}$.

We now define the creation operator $\varphi_{\xi \eta}^{* \xi^{\prime} \eta^{\prime}}(z)$ to be the map

$$
\mathcal{F}_{\xi \eta ; \tilde{\xi} \tilde{\eta}} \longrightarrow \mathcal{F}_{\xi^{\prime} \eta^{\prime} ; \tilde{\xi} \tilde{\eta}}, \quad f \mapsto \hat{\varphi}_{\xi \eta}^{* \xi^{\prime} \eta^{\prime}}(z) \circ f
$$

where the quasi-momentum $z$ should lie on the unit circle $|z|=1$. Likewise we define the annihilation operator $\varphi_{\xi \eta}^{\xi^{\prime} \eta^{\prime}}(z)$ to be the adjoint (with respect to (2.5)) of

$$
\mathcal{F}_{\xi \eta ; \tilde{\xi} \tilde{\eta}} \longrightarrow \mathcal{F}_{\xi \eta ; \tilde{\xi}^{\prime} \tilde{\eta}^{\prime}}, \quad f \mapsto f \circ \hat{\varphi}_{\tilde{\xi}^{\prime} \tilde{\eta}^{\prime}}^{\tilde{\tilde{\eta}} \tilde{}}(z)
$$

Unlike the case of the vertex models $[4,10]$, the particles in the RSOS models do not carry internal degees of freedom, or 'spin'.

It should be mentioned that we do not have a mathematical proof that $(3.5,3.6)$ have well defined meaning on the space of states. There are subtleties due to the fact that the type II VOs do not preserve the crystal structure [4]. One may also ask whether one can define operators analogous to $(3.3,3.4)$ by using other representations $V^{(l)}$ in place of $V^{(1)}$. Though such operators do exist mathematically, we suspect that they are not well defined in the above sense, cf. [10].

3.3 Momentum Using the definitions $(3.2,3.3,3.4)$ it is possible to calculate the momentum of the particles created by $\varphi_{\xi \eta}^{* \xi^{\prime} \eta^{\prime}}(z)$. The following commutation relation can be deduced from the $q \mathrm{KZ}$ equation (see [10]):

$$
\Phi_{\eta^{\prime}}^{\sigma\left(\eta^{\prime}\right) V^{(N)}}\left(z_{2}\right) \circ \Phi_{\eta}^{V^{(1) * a^{-1}} \eta^{\prime}}\left(z_{1}\right)=\tau\left(z_{1} / z_{2}\right)^{-1} \Phi_{\sigma(\eta)}^{V^{(1) * a-1}}{ }^{\sigma\left(\eta^{\prime}\right)}\left(z_{1}\right) \circ \Phi_{\eta}^{\sigma(\eta) V^{(N)}}\left(z_{2}\right),
$$


where

$$
\tau(z)=z^{-1 / 2} \frac{\Theta_{q^{4}}(q z)}{\Theta_{q^{4}}\left(q z^{-1}\right)} .
$$

It then follows from the definitions of the operators $T_{\eta \tilde{\eta}}^{\sigma(\eta) \sigma(\tilde{\eta})}$ and $\varphi_{\xi \eta}^{* \xi^{\prime} \eta^{\prime}}(z)$ that

$$
\widetilde{T}_{\eta^{\prime} \tilde{\eta}}\left(z_{2}\right) \circ \varphi_{\xi \eta}^{* \xi^{\prime} \eta^{\prime}}\left(z_{1}\right)=\tau\left(z_{1} / z_{2}\right)^{-2} \varphi_{\xi \eta}^{* \xi^{\prime} \eta^{\prime}}\left(z_{1}\right) \circ \widetilde{T}_{\eta \tilde{\eta}}\left(z_{2}\right)
$$

where $\widetilde{T}_{\eta \tilde{\eta}}(z)=T_{\sigma(\eta) \sigma(\tilde{\eta})}^{\eta \tilde{\eta}}(z) T_{\eta \tilde{\eta}}^{\sigma(\eta) \sigma(\tilde{\eta})}(z)$. (Note that $\sigma$ is an involution.) The formula for $\tau(z)$ is independent of the level $N$ and coincides with the momentum of quasi-particles in the vertex model of spin $N / 2$ [10]. On the other hand, in both the vertex and RSOS models the Hamiltonian can be obtained by differentiating the row transfer matrix with respect to the spectral parameter $z$. We thus conclude that the quasi-particles in the RSOS models carry the same energy and momentum as in the vertex models. However the particle structures are different because of the admissibility condition in the RSOS models.

3.4 Commutation relations The commutation relations of the operators $(3.3,3.4)$ can be deduced from those of the vertex operators as given in the Appendix 2. Restricting to $\left|z_{i}\right|=1$ we have

$$
\begin{aligned}
& \varphi_{\xi^{\prime} \eta^{\prime}}^{* \xi^{\prime \prime} \eta^{\prime \prime}}\left(z_{1}\right) \varphi_{\xi \eta}^{* \xi^{\prime} \eta^{\prime}}\left(z_{2}\right) \\
& =\sum_{\kappa, \zeta} \varphi_{\zeta \kappa}^{* \xi^{\prime \prime} \eta^{\prime \prime}}\left(z_{2}\right) \varphi_{\xi \eta}^{* \zeta \kappa}\left(z_{1}\right) \times W_{l}^{1}\left(\begin{array}{cc}
\xi & \xi^{\prime} \\
\zeta & \xi^{\prime \prime}
\end{array} \mid z_{1} / z_{2}\right) W_{N}^{* 1}\left(\begin{array}{cc}
\eta & \eta^{\prime} \\
\kappa & \eta^{\prime \prime}
\end{array} \mid z_{1} / z_{2}\right) .
\end{aligned}
$$

The Boltzmann weights are given by $(A 2.2)$. The $\varphi_{\xi \eta}^{\xi^{\prime} \eta^{\prime}}(z)$ enjoy the same commutation relations. As for $\varphi_{\xi \eta}^{\xi^{\prime} \eta^{\prime}}(z)$ and $\varphi_{\xi \eta}^{* \xi^{\prime} \eta^{\prime}}(z)$ we have

$$
\begin{aligned}
& \varphi_{\xi^{\prime} \eta^{\prime}}^{\xi^{\prime \prime} \eta^{\prime \prime}}\left(z_{1}\right) \varphi_{\xi \eta}^{* \xi^{\prime} \eta^{\prime}}\left(z_{2}\right) \\
& \quad=\sum_{\kappa, \zeta} \varphi_{\zeta \kappa}^{* \xi^{\prime \prime} \eta^{\prime \prime}}\left(z_{2}\right) \varphi_{\xi \eta}^{\zeta \kappa}\left(z_{1}\right) W_{l}^{1}\left(\begin{array}{cc}
\xi & \xi^{\prime} \\
\zeta & \xi^{\prime \prime}
\end{array} \mid q^{-2} z_{1} / z_{2}\right) W_{N}^{* 1}\left(\begin{array}{cc}
\eta & \eta^{\prime} \\
\kappa & \eta^{\prime \prime}
\end{array} \mid q^{-2} z_{1} / z_{2}\right) \\
& \times\left(K_{\xi}^{\zeta} K_{\eta}^{\kappa} / K_{\xi^{\prime}}^{\xi^{\prime \prime}} K_{\eta^{\prime}}^{\eta^{\prime \prime}}\right) q^{2 \Delta} \\
& +\delta_{\xi \xi^{\prime \prime}} \delta_{\eta \eta^{\prime \prime}} g_{\xi}^{\xi^{\prime}}(l, 1) \tilde{g}_{\eta}^{\eta^{\prime}}(N, 1) \delta\left(z_{1} / z_{2}\right),
\end{aligned}
$$

where $\Delta=\Delta_{\xi}+\Delta_{\xi^{\prime \prime}}+\Delta_{\eta}+\Delta_{\eta^{\prime \prime}}-\Delta_{\xi^{\prime}}-\Delta_{\zeta}-\Delta_{\eta^{\prime}}-\Delta_{\kappa}$. The delta function term comes from the simple pole of $(3.8)$ at $z_{1}=z_{2}$, and the forefactors $g_{\xi}^{\xi^{\prime}}(l, 1), \tilde{g}_{\eta}^{\eta^{\prime}}(N, 1)$ are given in Appendix 2, $(A 2.3, A 2.4)$.

The structure of the commutation relations (3.7) is precisely the same as that of the $S$-matrix for the deformation of the coset conformal field theory [15]. In fact the latter is reproduced from (3.7) in the limit $q \rightarrow 1$.

Acknowledgement. We wish to thank A. Le Clair, K. Miki, T. Nakashima and A. Nakayashiki for discussions. 


\section{Appendix 1.}

In this appendix, we will show how to get, in principle, the $q$-expansion of the eigenvectors of the RSOS model by means of the VO method. We will carry out the calculation to a few order in the Ising model, and see that it actually gives the correct expansion. See $[4,16]$ for a similar calculation in the spin-1/2 XXZ model.

A.1 Path expansions We call 'restricted paths' simply 'paths'. A path $p=(p(l))_{l \in \mathbf{Z}}$ is a sequence of integers, $p(l) \in S=\{0,1, \ldots, k\}$, with the admissibility condition (1.8). The 'Hilbert space' of the RSOS model is formally spanned by the paths: A 'state' is written as

$$
|v\rangle=\sum_{p} c(p)|p\rangle,
$$

where $c(p)$ is a formal power series in $q$. We may obtain the set of states of this form in two different ways:

(i) Perturbative diagonalization of the RSOS Hamiltonian.

(ii) Expansion of the states in $\mathcal{F}_{\xi \eta ;} \tilde{\xi} \tilde{\eta}$.

At $q=0$ the RSOS Hamiltonian is diagonal [12]. Therefore, the paths $|p\rangle$ are eigenvectors. The corresponding spectra are discrete, although they are continuous for $q \neq 0$. Therefore, the spectra are infinitely degenerate at $q=0$. In other words, the paths, which diagonalize the Hamiltonian at $q=0$, are mixed states of the $q \neq 0$ eigenvectors. Although we expect the eigenvectors in the form $(A 1.1)$, we do not know how to start the expansion. This is a difficulty in the approach (i).

For the lowest eigenvalue, this degeneracy is finite, and in fact, it is resolved by specifying the boundary conditions. Choosing an admissible pair $(b, c)$, we can calculate an expansion of the vacuum, the lowest eigenvector of the RSOS Hamiltonian, for which the sum $(A 1.1)$ is restricted to the paths satisfying $p(2 l)=\lambda_{b}$ and $p(2 l+1)=\lambda_{c}$ for large $|l|$.

We have another interpretation of (A1.1), i.e., (ii). Consider (2.2), and take a state $v$ in the subspace with a fixed $\lambda=\lambda_{a}$. We interprete this $a \in S$ to be the value of the state at the center of the one-dimensional lattice on which the RSOS Hamiltonian is defined. Namely, such $v$ is expanded in terms of $p$ satisfying $p(1)=a$. Moreover, the component $\mathcal{F}^{-}=\operatorname{Hom}_{U^{\prime}}(V(\tilde{\xi}) \otimes V(\tilde{\eta}), V(\lambda))\left(\operatorname{resp} . \mathcal{F}^{+}=\operatorname{Hom}_{U^{\prime}}(V(\lambda), V(\xi) \otimes V(\eta))\right)$ represents the right (resp. left) half of the restricted paths: A vector $v^{ \pm}$in $\mathcal{F}^{ \pm}$has an expansion

$$
\left|v^{ \pm}\right\rangle=\sum_{p^{ \pm}} c\left(v^{ \pm} ; p^{ \pm}\right)\left|p^{ \pm}\right\rangle,
$$

where $p^{+}=\left(p^{+}(l)\right)_{l \geq 1}\left(\right.$ or $\left.p^{-}=\left(p^{-}(l)\right)_{l \leq 1}\right)$ satisfies $p^{ \pm}(1)=\lambda$ and the appropriate boundary condition for large $|l|$.

Let us consider $\mathcal{F}^{+}$. The coefficients $c\left(p^{+}\right)$'s in $(A 1.2)$ are determined from $v^{+}$as follows. Induce a map

$$
\Phi_{a(l), \ldots, a(1)}^{\xi \eta}: \operatorname{Hom}_{U^{\prime}}\left(V\left(\lambda_{a(1)}\right), V(\xi) \otimes V(\eta)\right) \rightarrow \operatorname{Hom}_{U^{\prime}}\left(V\left(\lambda_{a(l)}\right), V(\xi) \otimes V\left(\sigma^{l-1} \eta\right)\right)
$$

from (2.4): For $v^{+}$in

$$
\begin{aligned}
& \operatorname{Hom}_{U^{\prime}}\left(V\left(\lambda_{a(1)}\right), V(\xi) \otimes V(\eta)\right) \\
& \simeq \oplus_{\lambda^{\prime}} \operatorname{Hom}_{U^{\prime}}\left(V\left(\lambda^{\prime}\right), V(\xi) \otimes V\left(\sigma^{l-1} \eta\right)\right) \otimes \operatorname{Hom}_{U^{\prime}}\left(V\left(\lambda_{a(1)}\right), V\left(\lambda^{\prime}\right) \otimes V^{\otimes(l-1)}\right)
\end{aligned}
$$


we have

$$
v^{+}=\sum_{a(l), \ldots, a(2)} \Phi_{a(l), \ldots, a(1)}^{\xi \eta}\left(v^{+}\right) \otimes \Phi_{\lambda_{a(l-1)}}^{\lambda_{a(l)} V} \circ \cdots \circ \Phi_{\lambda_{a(1)}}^{\lambda_{a(2)} V} .
$$

We consider $\Phi_{p(l), \ldots, p(1)}^{\xi \eta}$. If $l$ is large enough, we have $\lambda_{p^{+}(l)}=\xi+\sigma^{l-1} \eta$. Therefore, we can define $c^{(l)}\left(v^{+} ; p^{+}\right)$to be the coefficient of $u_{\xi} \otimes u_{\sigma^{l-1} \eta}$ considered as an element of $\operatorname{Hom}_{U^{\prime}}\left(V\left(\lambda_{p^{+}(l)}\right), V(\xi) \otimes V\left(\sigma^{l-1} \eta\right)\right) \simeq \Omega_{\xi \sigma^{l-1} \eta \lambda_{a(l)}} \subset V(\xi) \otimes V\left(\sigma^{l-1} \eta\right)$, in the expansion of $\Phi_{p^{+}(l), \ldots, p^{+}(1)}^{\xi \eta}\left(v^{+}\right)$. Then, we set

$$
c\left(p^{+}\right)=\lim _{l \rightarrow \infty} \frac{c^{(l)}\left(v^{+} ; p^{+}\right)}{c^{(l)}\left(u_{\xi} \otimes u_{\eta} ; p_{\xi \eta}^{+}\right)},
$$

where $p_{\xi \eta}^{+}=\left(\begin{array}{lll}\cdots & a_{1} a_{0} a_{1} a_{0}\end{array}\right)$ such that $\lambda_{a_{0}}=\xi+\eta$ and $\lambda_{a_{1}}=\xi+\sigma \eta$.

The right half is similar. Therefore, given a state $v$ in (2.2), we can expand it in the form $(A 1.1)$. We wish to check the equality of (i) and (ii), e.g., for the vacuum state. Or, a similar check is the following. We consider the CTM Hamiltonian. As in [16], we expect the equality between the following.

(ia) The perturbative diagonalization of the CTM Hamiltonian.

(iia) The path expansion of $\operatorname{Hom}_{U^{\prime}}(V(\lambda), V(\xi) \otimes V(\eta))$ described above.

In the following section we report on our check for the lowest eigenvector of the Ising CTM Hamiltonian versus the highest weight vector $u_{\Lambda_{i}} \otimes u_{\Lambda_{j}} \in \operatorname{Hom}_{U^{\prime}}\left(V\left(\Lambda_{i}+\right.\right.$ $\left.\left.\Lambda_{j}\right), V\left(\Lambda_{i}\right) \otimes V\left(\Lambda_{j}\right)\right)$.

A.2 The lowest eigenvector of the Ising CTM Hamiltonian If $l=N=1$, the RSOS model reduces to two non-interacting Ising models. The set $S$ is $\{0,1,2\}$. We identify 0 with the + Ising-spin and 2 with the - Ising-spin. We write the Ising spin operator by $\sigma_{l}^{x, y, z}$. The CTM Hamiltonians are

$$
\begin{aligned}
& B_{\text {even }}=-C_{z} \sum_{l \geq 1}(2 l-1)\left(\sigma_{2 l+1}^{z} \sigma_{2 l-1}^{z}-1\right)+C_{x} \sum_{l \geq 2} 2(l-1) \sigma_{2 l-1}^{x}+R_{\text {even }}, \\
& B_{\text {odd }}=-C_{z} \sum_{l \geq 1} 2 l\left(\sigma_{2 l+2}^{z} \sigma_{2 l}^{z}-1\right)+C_{x} \sum_{l \geq 1}(2 l-1) \sigma_{2 l}^{x}+R_{\text {odd }},
\end{aligned}
$$

where

$$
\begin{aligned}
& C_{z}=\frac{1}{4}-\frac{\sum_{n \in \mathbf{Z}}(-)^{n} n q^{2 n(2 n-1)}}{\sum_{n \in \mathbf{Z}}(-)^{n} q^{2 n(2 n-1)}}=\frac{1}{4}+q^{2}+q^{4}+\cdots, \\
& C_{x}=q \frac{\sum_{n \in \mathbf{Z}}(-)^{n-1} n q^{4 n(n-1)}}{\sum_{n \in \mathbf{Z}}(-)^{n} q^{4 n^{2}}}=q+2 q^{5}+\cdots,
\end{aligned}
$$

and $R_{\text {even/odd }}$ is the renormalization constant to be determined. The operator $B_{\text {even }}$ corresponds to the boundary condition $(\xi, \eta)=\left(\Lambda_{0}, \Lambda_{0}\right)$ (or $(\xi, \eta)=\left(\Lambda_{1}, \Lambda_{1}\right)$ ). The operator $B_{\text {odd }}$ corresponds to the boundary condition $(\xi, \eta)=\left(\Lambda_{0}, \Lambda_{1}\right)$ (or $(\xi, \eta)=$ $\left.\left(\Lambda_{1}, \Lambda_{0}\right)\right)$. Without loss of generality, we restrict to the case $\xi=\Lambda_{0}$. It means at $q=0$ the lowest eigenvector reduces to a single path: ( $\cdots 121212$ ) (for $B_{\text {even }}$ ) or $(\cdots 212121)$ (for $B_{\text {odd }}$ ). In the expansion of the lowest eigenvectors, the rightmost spins, i.e., 2 in the former or 1 in the latter, are kept fixed. The admissibility condition 
also freezes all the spins of the value 1 . If we neglect the 1's, and only keep writing the Ising spins $-/+$ for $2 / 0$, both cases are simply $(\cdots---)$. Let us use abbreviated symbols: e.g.,

$$
\begin{aligned}
& |\phi\rangle=(\cdots---) \\
& |1\rangle=(\cdots--+) \\
& |2\rangle=(\cdots-+-) \\
& |2,1\rangle=(\cdots-++)
\end{aligned}
$$
take

We determine $R_{\text {even/odd }}$ and calculate the lowest eigenvector $|v a c\rangle=\sum c(p)|p\rangle$. We

$$
R_{\text {even }}=\sum_{l \geq 2} 2(l-1) R_{2(l-1)} \quad \text { and } \quad R_{\text {odd }}=\sum_{l \geq 1}(2 l-1) R_{2 l-1},
$$

where $R_{l}$ should be chosen so that the lowest eigenvalue is zero. The condition $B|v a c\rangle=$ 0 gives us a way to calculate $c(p)$ and $R$. Taking the coefficient of $|\phi\rangle$ in $B|v a c\rangle=$ $\sum c(p) B|p\rangle$, we have

$$
\begin{aligned}
& \sum_{l \geq 2} 2(l-1)\left(C_{x} c(|l\rangle)+R_{2(l-1)}\right)=0, \quad \text { for } B_{\text {even }} \\
& \sum_{l \geq 1}(2 l-1)\left(C_{x} c(|l\rangle)+R_{2 l-1}\right)=0, \quad \text { for } B_{\text {odd }} .
\end{aligned}
$$

Therefore we choose

$$
\begin{aligned}
& R_{2(l-1)}=-C_{x} c(|l\rangle), \quad l \geq 2, \\
& R_{2 l-1}=-C_{x} c(|l\rangle), \quad l \geq 1 .
\end{aligned}
$$

We assume that $c(p)$ is expanded in $q$ as

$$
c(p)=\sum_{i \geq 0} q^{m+2 i} c_{2 i}(p), \quad \text { for } p=\left|j_{m}, j_{m-1}, \ldots, j_{1}\right\rangle .
$$

On this assumption, the coefficient $c(p)$ is successively and consistently determined in the form of power series of $q$ by using the equation $B|v a c\rangle=0$ expanded in $q$. Let us give the path expansions of the lowest eigenvectors with the coefficient of $|\phi\rangle$ normalized to 1 . Keeping the paths $\left|j_{m}, j_{m-1}, \ldots, j_{1}\right\rangle$ satisfying $m \leq 3$ and the coefficients up to $q^{5}$, we have

$$
\begin{aligned}
& |v a c\rangle_{\text {even }}=|\phi\rangle-\left(q-3 q^{3}+6 q^{5}\right) \sum_{m \geq 2}|m\rangle-q^{5}|2\rangle \\
& +\left(q^{2}-6 q^{4}\right)\left(\sum_{m>l \geq 2}|m, l\rangle+\sum_{m \geq 2}|m+1, m\rangle\right)+q^{4} \sum_{m \geq 2}|m+2, m\rangle \\
& \quad-\left(q^{3}-9 q^{5}\right)\left(\sum_{m>l>k \geq 2}|m, l, k\rangle+\sum_{m>l+1, l \geq 2}|m, l+1, l\rangle+\sum_{m>l \geq 2}|m+1, m, l\rangle\right. \\
& \left.\quad+2 \sum_{m \geq 2}|m+2, m+1, m\rangle\right)+q^{5}\left(\sum_{m \geq 2}|m+2, m+1, m\rangle-\sum_{m>l+2, l \geq 2}|m, l+2, l\rangle\right.
\end{aligned}
$$




$$
\begin{aligned}
& \left.-\sum_{m>l \geq 2}|m+2, m, l\rangle-3 \sum_{m \geq 2}(|m+3, m+1, m\rangle+|m+3, m+2, m\rangle)\right)+\cdots, \\
& |v a c\rangle_{\text {odd }}=|\phi\rangle-\left(q-3 q^{3}+6 q^{5}\right) \sum_{m \geq 1}|m\rangle-\left(q^{3}-3 q^{5}\right)|1\rangle-q^{5}|2\rangle \\
& +\left(q^{2}-6 q^{4}\right)\left(\sum_{m>l \geq 1}|m, l\rangle+\sum_{m \geq 1}|m+1, m\rangle\right)+q^{4}\left(2|2,1\rangle+\sum_{m>1}|m, 1\rangle+\sum_{m \geq 1}|m+2, m\rangle\right) \\
& -\left(q^{3}-9 q^{5}\right)\left(\sum_{m>l>k \geq 1}|m, l, k\rangle+\sum_{m>l+1, l \geq 1}|m, l+1, l\rangle+\sum_{m>l \geq 1}|m+1, m, l\rangle\right. \\
& \left.+2 \sum_{m \geq 1}|m+2, m+1, m\rangle\right)+q^{5}\left(\sum_{m \geq 1}|m+2, m+1, m\rangle-\sum_{m>l+2, l \geq 1}|m, l+2, l\rangle\right. \\
& -\sum_{m>l \geq 1}|m+2, m, l\rangle-3 \sum_{m \geq 1}(|m+3, m+1, m\rangle+|m+3, m+2, m\rangle) \\
& \left.-5|3,2,1\rangle-2 \sum_{m>2}|m, 2,1\rangle-\sum_{m>l>1}|m, l, 1\rangle-\sum_{m>1}|m+1, m, 1\rangle\right)+\cdots
\end{aligned}
$$

A.3 Expansion of $u_{\Lambda_{0}} \otimes u_{\Lambda_{i}}$ We use the following vectors in $V\left(\Lambda_{0}\right)$.

$$
\begin{aligned}
& v_{1}=u_{\Lambda_{0}}, \quad v_{2}=f_{0} v_{1}, \quad v_{3}=\frac{1}{[2]} f_{1} v_{2}, \quad v_{4}=f_{0} v_{3}, \\
& v_{5}=f_{1} v_{3}, \quad v_{6}=\frac{1}{[2]([3]-1)}\left([3] f_{1} v_{4}-f_{0} v_{5}\right), \quad v_{7}=\frac{1}{[3]-1}\left(f_{0} v_{5}-f_{1} v_{4}\right) .
\end{aligned}
$$

By $v_{i}^{\prime}(1 \leq i \leq 7)$ we mean the vectors in $V\left(\Lambda_{1}\right)$ that are the images of $v_{i}(1 \leq i \leq 7)$ by the Dynkin diagram automorphism.

The first few highest weight vectors in $V\left(\Lambda_{0}\right) \otimes V\left(\Lambda_{0}\right)$ are as follows.

$$
\begin{aligned}
& w_{1}=v_{1} \otimes v_{1} \\
& w_{2}=v_{1} \otimes v_{2}-q v_{2} \otimes v_{1} \\
& w_{3}=v_{1} \otimes v_{4}-q v_{2} \otimes v_{3}+q^{3} v_{3} \otimes v_{2}-q^{4} v_{4} \otimes v_{1} \\
& w_{4}=v_{1} \otimes v_{7}-q v_{2} \otimes v_{5}+q^{3}[2] v_{3} \otimes v_{3}-q^{3} v_{5} \otimes v_{2}+q^{6} v_{7} \otimes v_{1}
\end{aligned}
$$

Similarly, we have highest weight vectors in $V\left(\Lambda_{0}\right) \otimes V\left(\Lambda_{1}\right)$.

$$
\begin{aligned}
& w_{1}^{\prime}=v_{1} \otimes v_{1}^{\prime} \\
& w_{2}^{\prime}=v_{1} \otimes v_{3}^{\prime}-q v_{2} \otimes v_{2}^{\prime}+q^{3} v_{3} \otimes v_{1}^{\prime} \\
& w_{3}^{\prime}=v_{1} \otimes v_{6}^{\prime}-q v_{2} \otimes v_{4}^{\prime}+q^{3} v_{3} \otimes v_{3}^{\prime}-q^{4} v_{4} \otimes v_{2}^{\prime}+q^{6} v_{6} \otimes v_{1}^{\prime}
\end{aligned}
$$

Their weights are

$$
\begin{array}{ccccccc}
w_{1} & w_{2} & w_{3} & w_{4} & w_{1}^{\prime} & w_{2}^{\prime} & w_{3}^{\prime} \\
2 \Lambda_{0} & 2 \Lambda_{1}-\delta & 2 \Lambda_{1}-2 \delta & 2 \Lambda_{0}-2 \delta & \Lambda_{0}+\Lambda_{1} & \Lambda_{0}+\Lambda_{1}-\delta & \Lambda_{0}+\Lambda_{1}-2 \delta
\end{array}
$$

The vectors $w_{1}$ and $w_{4}$ are considered as vectors in $\operatorname{Hom}_{U^{\prime}}\left(V\left(2 \Lambda_{0}\right), V\left(\Lambda_{0}\right) \otimes V\left(\Lambda_{0}\right)\right), w_{2}$ and $w_{3}$ in $\operatorname{Hom}_{U^{\prime}}\left(V\left(2 \Lambda_{1}\right), V\left(\Lambda_{0}\right) \otimes V\left(\Lambda_{0}\right)\right)$, and the rest in $\operatorname{Hom}_{U^{\prime}}\left(V\left(\Lambda_{0}+\Lambda_{1}\right), V\left(\Lambda_{0}\right) \otimes\right.$ $\left.V\left(\Lambda_{1}\right)\right)$. 
By using these vectors, we can write down a few terms in the application of $\Phi_{a(2), a(1)}$ given in $(A 1.3)$ :

$$
\begin{aligned}
& \Phi_{12}^{\Lambda_{0} \Lambda_{0}}\left(w_{1}\right)=w_{1}^{\prime}+\frac{1}{[4]} w_{2}^{\prime}+\frac{[3]}{[8][2]} w_{3}^{\prime}+\cdots, \\
& \Phi_{10}^{\Lambda_{0} \Lambda_{0}}\left(w_{2}\right)=w_{1}^{\prime}-\frac{[3]}{[4]} w_{2}^{\prime}-\frac{[5]}{[8][2]} w_{3}^{\prime}+\cdots, \\
& \Phi_{10}^{\Lambda_{0} \Lambda_{0}}\left(w_{3}\right)=\frac{1}{[2]} w_{1}^{\prime}+\frac{[5]}{[4][2]} w_{2}^{\prime}-\frac{[7][3]}{[8][2]^{2}} w_{3}^{\prime}+\cdots, \\
& \Phi_{12}^{\Lambda_{0} \Lambda_{0}}\left(w_{4}\right)=-\frac{1}{[4]} w_{1}^{\prime}+\frac{[5][3]}{[4]^{2}} w_{2}^{\prime}-\frac{[7][5]}{[8][4][2]} w_{3}^{\prime}+\cdots, \\
& \Phi_{01}^{\Lambda_{0} \Lambda_{1}}\left(w_{1}^{\prime}\right)=-\frac{1}{[2]} w_{2}-\frac{[3]}{[6][2]} w_{3}+\cdots, \\
& \Phi_{01}^{\Lambda_{0} \Lambda_{1}}\left(w_{2}^{\prime}\right)=\frac{[3]}{[2]^{2}} w_{2}-\frac{[5][3]}{[6][2]^{2}} w_{3}+\cdots, \\
& \Phi_{01}^{\Lambda_{0} \Lambda_{1}}\left(w_{3}^{\prime}\right)=\frac{[5]}{[4][2]^{2}} w_{2}+\frac{[7][3]^{2}}{[6][4][2]^{2}} w_{3}+\cdots, \\
& \Phi_{21}^{\Lambda_{0} \Lambda_{1}}\left(w_{1}^{\prime}\right)=w_{1}-\frac{[3]}{[6][4][2]} w_{4}+\cdots, \\
& \Phi_{21}^{\Lambda_{0} \Lambda_{1}}\left(w_{2}^{\prime}\right)=\frac{1}{[2]} w_{1}+\frac{[5][3]^{2}}{[6][4][2]^{2}} w_{4}+\cdots, \\
& \Phi_{21}^{\Lambda_{0} \Lambda_{1}}\left(w_{3}^{\prime}\right)=\frac{[3]}{[4][2]} w_{1}-\frac{[7][5][3]}{[6][4]^{2}[2]^{2}} w_{4}+\cdots
\end{aligned}
$$

Using these data we can calculate $c^{(l)}\left(v^{+} ; p^{+}\right)$. As an example let us show how the calculation goes for $v^{+}=w_{1}$ and $p^{+}=p_{\Lambda_{0} \Lambda_{0}}^{+}$. The operator $\Phi_{p^{+}(l), \cdots, p^{+}(1)}^{\xi \eta}$ is given by the composition $\Phi_{p^{+}(l), p^{+}(l-1)}^{\xi \sigma^{l} \eta} \circ \cdots \circ \Phi_{p^{+}(2), p^{+}(1)}^{\xi \eta}$. Applying these operators to $w_{1}$, we get up to $q^{6}$

$$
\begin{aligned}
& \Phi_{12}^{\Lambda_{0} \Lambda_{0}}\left(w_{1}\right)=w_{1}^{\prime}+\left(q^{3}-q^{5}\right) w_{2}^{\prime}+q^{6} w_{3}^{\prime}+\cdots, \\
& \Phi_{21}^{\Lambda_{0} \Lambda_{1}} \circ \Phi_{12}^{\Lambda_{0} \Lambda_{0}}\left(w_{1}\right)=\left(1+q^{4}-2 q^{6}\right) w_{1}+q^{5} w_{4}+\cdots, \\
& \Phi_{12}^{\Lambda_{0} \Lambda_{0}} \circ \Phi_{21}^{\Lambda_{0} \Lambda_{1}} \circ \Phi_{12}^{\Lambda_{0} \Lambda_{0}}\left(w_{1}\right)=\left(1+q^{4}-2 q^{6}\right) w_{1}^{\prime}+q^{3} w_{2}^{\prime}+\cdots, \\
& \Phi_{21}^{\Lambda_{0} \Lambda_{1}} \circ \Phi_{12}^{\Lambda_{0} \Lambda_{0}} \circ \Phi_{21}^{\Lambda_{0} \Lambda_{1}} \circ \Phi_{12}^{\Lambda_{0} \Lambda_{0}}\left(w_{1}\right)=\left(1+2 q^{4}-3 q^{6}\right) w_{1}+q^{5} w_{4}+\cdots, \\
& \Phi_{12}^{\Lambda_{0} \Lambda_{0}} \circ \Phi_{21}^{\Lambda_{0} \Lambda_{1}} \circ \Phi_{12}^{\Lambda_{0} \Lambda_{0}} \circ \Phi_{21}^{\Lambda_{0} \Lambda_{1}} \circ \Phi_{12}^{\Lambda_{0} \Lambda_{0}}\left(w_{1}\right)=\left(1+2 q^{4}-3 q^{6}\right) w_{1}^{\prime}+q^{3} w_{2}^{\prime}+\cdots .
\end{aligned}
$$

The coefficient of $w_{1}$ (or $w_{1}^{\prime}$ ) in the above expansion of $\Phi_{p^{+}(l), \cdots, p^{+}(1)}\left(w_{1}\right)$ gives $c^{(l)}\left(w_{1} ; p_{\Lambda_{0} \Lambda_{0}}^{+}\right)$, that is, for $l \geq 3$

$$
c^{(l)}\left(w_{1} ; p_{\Lambda_{0} \Lambda_{0}}^{+}\right)=1+L q^{4}-(L+1) q^{6}+\cdots
$$

where $L=l / 2-1$ for $l$ :even (or $(l-1) / 2$ for $l$ :odd). In a similar way, applying $\Phi_{p^{+}(l), p^{+}(l-1)}^{\xi \sigma^{l} \eta} \circ \cdots \circ \Phi_{p^{+}(2), p^{+}(1)}^{\xi \eta}$ to $w_{1}\left(\right.$ or $\left.w_{1}^{\prime}\right)$, we can calculate $c^{(l)}\left(w_{1} ; p^{+}\right)\left(\right.$or $\left.c^{(l)}\left(w_{1}^{\prime} ; p^{+}\right)\right)$ 
up to $q^{5}$ for the paths $p^{+}=|m\rangle,|n, m\rangle$ and $|m+2, m+1, m\rangle$ for $n>m \geq 2$ (or $n>m \geq 1$ ). The results coincide with those obtained by the perturbative calculation of $|v a c\rangle$ in A.2. Therefore it is strongly suggested that the equality holds between the perturbative diagonalization of the CTM Hamiltonian and the path expansion of the states in $\mathcal{F}^{+}$.

\section{Appendix 2. Connection coefficients}

Here we include the explicit form of the connection coefficients in the case $N=1$ [3]. Fix $\lambda \in P_{k}^{0}$, and set

$$
\begin{aligned}
& p=q^{2(k+2)}, \quad s=\frac{1}{2(k+2)}, \quad \lambda_{ \pm}=\lambda \pm \bar{\Lambda}_{1}, \quad r_{+}=1-r_{-}, \quad r_{-}=\Delta_{\lambda_{+}}-\Delta_{\lambda_{-}} \\
& (z ; p)_{\infty}=\prod_{j \geq 0}\left(1-z p^{j}\right), \quad\left(z ; p, p^{\prime}\right)_{\infty}=\prod_{i, j \geq 0}\left(1-z p^{i} p^{\prime j}\right) \\
& \Theta_{p}(z)=(z ; p)_{\infty}(p / z ; p)_{\infty}(p ; p)_{\infty}, \quad \Gamma_{p}(x)=\frac{(p ; p)_{\infty}}{\left(p^{x} ; p\right)_{\infty}}(1-p)^{1-x} \\
& \psi(z)=\frac{\left(q^{4} z ; q^{4}, p\right)_{\infty}\left(z ; q^{4}, p\right)_{\infty}}{\left(q^{2} z ; q^{4}, p\right)_{\infty}^{2}}
\end{aligned}
$$

Let $V=V^{(1)}$ and let $\bar{R}_{11}(z)=\bar{R}(z)$ be as in [4], eq.(6.16). Then

$$
\begin{aligned}
& \bar{R}\left(z_{1} / z_{2}\right) \Phi_{\mu}^{\nu V_{1}}\left(z_{1}\right) \Phi_{\lambda}^{\mu V_{2}}\left(z_{2}\right) \\
& \quad=\sum_{\mu^{\prime}} \Phi_{\mu^{\prime}}^{\nu V_{2}}\left(z_{2}\right) \Phi_{\lambda}^{\mu^{\prime} V_{1}}\left(z_{1}\right) W_{k}^{1}\left(\begin{array}{cc}
\lambda & \mu \\
\mu^{\prime} & \nu
\end{array} \mid z_{1} / z_{2}\right) \\
& \left(\bar{R}\left(z_{1} / z_{2}\right)^{t}\right)^{-1} \Phi_{\mu}^{V_{1}^{* a-1} \nu}\left(z_{1}\right) \Phi_{\lambda}^{V^{* a}{ }^{-1}}{ }^{\prime}\left(z_{2}\right) \\
& \quad=\sum_{\mu^{\prime}} \Phi_{\mu^{\prime}}^{V_{2}^{* a-1} \nu}\left(z_{2}\right) \Phi_{\lambda}^{V_{1}^{* a-1} \mu^{\prime}}\left(z_{1}\right) W_{k}^{* 1}\left(\begin{array}{cc}
\lambda & \mu \\
\mu^{\prime} & \nu
\end{array} \mid z_{1} / z_{2}\right),
\end{aligned}
$$

where

$$
\begin{aligned}
\left.W_{k}^{1}\left(\begin{array}{cc}
\lambda & \mu \\
\mu^{\prime} & \nu
\end{array}\right) z\right) & =\bar{W}_{k}^{1}\left(\begin{array}{cc}
\lambda & \mu \\
\mu^{\prime} & \nu
\end{array}\right) \times \frac{\psi\left(p z^{-1}\right)}{\psi(p z)} z^{\Delta_{\lambda}+\Delta_{\nu}-\Delta_{\mu}-\Delta_{\mu^{\prime}}} \\
\left.W_{k}^{* 1}\left(\begin{array}{cc}
\lambda & \mu \\
\mu^{\prime} & \nu
\end{array}\right) z\right) & =\bar{W}_{k}^{1}\left(\begin{array}{cc}
\lambda & \mu \\
\mu^{\prime} & \nu
\end{array}\right) \times \frac{\psi\left(z^{-1}\right)}{\psi(z)} z^{\Delta_{\lambda}+\Delta_{\nu}-\Delta_{\mu}-\Delta_{\mu^{\prime}}}
\end{aligned}
$$

and

$$
\begin{aligned}
& \bar{W}_{k}^{1}\left(\begin{array}{cc}
\lambda & \mu \\
\mu & \nu
\end{array}\right)=1 \quad\left(\mu-\lambda=\nu-\mu= \pm \bar{\Lambda}_{1}\right), \\
& \left.\bar{W}_{k}^{1}\left(\begin{array}{cc}
\lambda & \lambda_{ \pm} \\
\lambda_{ \pm} & \lambda
\end{array}\right) z\right)=\frac{\Theta_{p}\left(q^{2}\right) \Theta_{p}\left(p^{r_{ \pm}} z\right)}{\Theta_{p}\left(p^{r_{ \pm}}\right) \Theta_{p}\left(q^{2} z\right)} z^{(1 \pm 1) / 2}, \\
& \bar{W}_{k}^{1}\left(\begin{array}{cc}
\lambda & \lambda_{\mp} \\
\lambda_{ \pm} & \lambda
\end{array}\right)=q \frac{\Gamma_{p}\left(r_{ \pm}\right) \Gamma_{p}\left(r_{ \pm}\right)}{\Gamma_{p}\left(2 s+r_{ \pm}\right) \Gamma_{p}\left(-2 s+r_{ \pm}\right)} \frac{\Theta_{p}(z)}{\Theta_{p}\left(q^{2} z\right)} .
\end{aligned}
$$


Up to a 'gauge' they coincide with the Boltzmann weights due to Andrews-BaxterForrester [11]. For general $N$ the weights (1.14) are constructed from $(A 2.2)$ through the fusion procedure [6].

The following formulas are used in Sect. 3:

$$
\begin{aligned}
g_{\lambda}^{\lambda_{ \pm}}(k, 1) & =\left\langle u_{\lambda}\left|\widetilde{\Phi}_{\lambda_{ \pm} V^{(1)}}^{\lambda}(z) \widetilde{\Phi}_{\lambda}^{\lambda_{ \pm} V^{(1)}}(z)\right| u_{\lambda}\right\rangle \\
& =\frac{\left(p q^{2} ; q^{4}, p\right)_{\infty}^{2}}{\left(p ; q^{4}, p\right)_{\infty}\left(p q^{4} ; q^{4}, p\right)_{\infty}} \frac{\left(p^{r_{ \pm}} q^{2} ; p\right)_{\infty}}{\left(p^{r_{ \pm}} ; p\right)_{\infty}}, \\
\tilde{g}_{\lambda}^{\lambda_{ \pm}}(k, 1) & =\operatorname{Res}_{z_{1}=z_{2}}\left\langle u_{\lambda}\left|\widetilde{\Phi}_{\lambda_{ \pm}}^{V^{(1)}} \lambda\left(z_{1}\right) \widetilde{\Phi}_{V^{(1)} \lambda}^{\lambda_{ \pm}}\left(z_{2}\right)\right| u_{\lambda}\right\rangle d\left(z_{1} / z_{2}\right) \\
& =\frac{\left(q^{2} ; q^{4}, p\right)_{\infty}^{2}}{\left(q^{4} ; q^{4}, p\right)_{\infty}^{2}(p ; p)_{\infty}} \frac{\left(p^{r_{ \pm}} q^{2} ; p\right)_{\infty}}{\left(p^{r_{ \pm}} ; p\right)_{\infty}} .
\end{aligned}
$$

\section{References}

[1] see e.g. Jimbo M, Introduction to the Yang-Baxter equation, Int. J. Mod. Phys. A, 4, (1989) 3759-3777.

[2] Bazhanov V V and Stroganov Yu G, Chiral Potts model as a descendant of the six vertex models, J. Stat. Phys., 51, (1990) 799-817.

[3] Frenkel I B and Reshetikhin N Yu, Quantum affine algebras and holonomic difference equations, Commun. Math. Phys., 146, (1992) 1-60.

[4] Davies B, Foda O, Jimbo M, Miwa T and Nakayashiki A, Diagonalization of the XXZ Hamiltonian by vertex operators, RIMS preprint, 873, (1992).

[5] Baxter R J, Exactly solved models in statistical mechanics, Academic Press, London 1982.

[6] Date E, Jimbo M, Kuniba A, Miwa T and Okado M, Exactly solvable SOS models: Local height probabilities and theta function identities, Nucl. Phys. B, 290, [FS20] (1987) 231-273.

[7] Date, E., Jimbo, M., Kuniba, A., Miwa, T. and Okado, M, One dimensional configuration sums in vertex models and affine Lie algebra characters, Lett. Math. Phys., 17, (1989) 69-77.

[8] Kang S-J, Kashiwara M, Misra K, Miwa T, Nakashima T and Nakayashiki A, Affine crystals and vertex models, Int. J. Mod. Phys. A, 7, Suppl. 1A (1992) 449-484.

[9] Jimbo M, Miki K, Miwa T and Nakayashiki A, Correlation functions of the XXZ model for $\Delta<-1$, RIMS preprint, 877, (1992).

[10] Idzumi M, Iohara K, Jimbo M, Miwa T, Nakashima T and Tokihiro T, Quantum affine symmetry in vertex models, RIMS preprint, (1992).

[11] Andrews G E, Baxter R J and Forrester P J, Eight-vertex SOS model and generalized Rogers-Ramanujan-type identities, J. Stat. Phys., 35, (1984) 193-266.

[12] Date E, Jimbo M and Okado M, Crystal base and $q$ vertex operators, Osaka Univ. Math. Sci. preprint, 1, (1991).

[13] Smirnov F A, Dynamical symmetries of massive integrable models, Int. J. Mod. Phys. A, 7, Suppl. 1B (1992) 813-837, 839-858.

[14] Bernard D, Hidden Yangians in 2D massive current algebras, Commun. Math. Phys., 137, (1991) 191-208.

[15] Ahn C, Bernard D and LeClair A, Fractional supersymmetries in perturbed coset CFT's and integrable soliton theory, Nucl. Phys., B340, (1990) 721.

[16] Foda $\mathrm{O}$ and Miwa $\mathrm{T}$, Corner transfer matrices and quantum affine algebras, Int. J. Mod. Phys. A, 7, Suppl. 1A (1992) 1-53. 